\title{
A Dislocation Mechanism for Cryogenic Relaxations in Crystalline Polymers
}

\author{
Anne Hiltner and Eric Baer \\ Division of Macromolecular Science, Case Western Reserve \\ University, Cleveland, Ohio 44106, U.S.A.
}

(Received September 28, 1971)

\begin{abstract}
The experimental properties of the $\delta_{\mathrm{c}}$-relaxation observed in polyethylene, poly(oxymethylene), and poly(ethylene terephthalate) at about $50^{\circ} \mathrm{K}$ are reviewed. A mechanism is proposed involving the thermally activated redistribution of kinks along a discontinuous dislocation under an applied stress. The activation energy and relaxation intensity are discussed with reference to specific chain conformations in the dislocation. It is suggested that the kinked dislocations arise from external stresses transferred to the crystalline or ordered regions of the polymer possibly via tie molecules. These stresses are greatest in deformed material or material annealed under constraint and accounts for the increase in peak height observed in these specimens.

KEY WORDS Cryogenics / Dislocation / Polyethylene / Poly(ethylene terephthalate) / Poly(oxymethylene) / $\delta$-Relaxation / Kink / Conformation /
\end{abstract}

Secondary relaxations above $77^{\circ} \mathrm{K}$ have been observed in many crystalline polymers and the effects of orientation and crystallinity on the relaxation spectrum have been studied by various investigators. Their results are reviewed in the excellent monograph by McCrum, Williams, and Read. ${ }^{1}$ The possibility that linear, crystalline polymers might show relaxation processes below $77^{\circ} \mathrm{K}$ has received less attention. Early investigators reported no relaxation processes below $77^{\circ} \mathrm{K}$ in polyethylene ${ }^{2}$ and poly(tetrafluoroethylene). ${ }^{3} \quad$ However, with the use of more sensitive experimental techniques, very low-temperature relaxations have been observed in this laboratory in a number of linear polymers. Moreover these relaxations were found to be very sensitive to the changes in solid-state structure caused by annealing or deformation. Speculation concerning the mechanism responsible for these relaxation processes has been hampered by the lack of experimental information, and only recently has sufficient data become available to warrant a detailed consideration of the underlying mechanism.

\section{CRYOGENIC RELAXATIONS IN POLYMERS}

Dynamic mechanical relaxations in polymers in the temperature range 4.2 to $77^{\circ} \mathrm{K}$ have been reported by a number of authors. Previous workers however have been primarily concerned with polymers containing side groups, and relaxations observed below $100^{\circ} \mathrm{K}$ were attributed to motions of the pendent group. ${ }^{2-7}$ This assignment has been substantiated for the $\delta$-peak in polystyrene (PS) by correlation of mechanical, NMR, and dielectric measurements of polystyrene and a variety of halogenated and

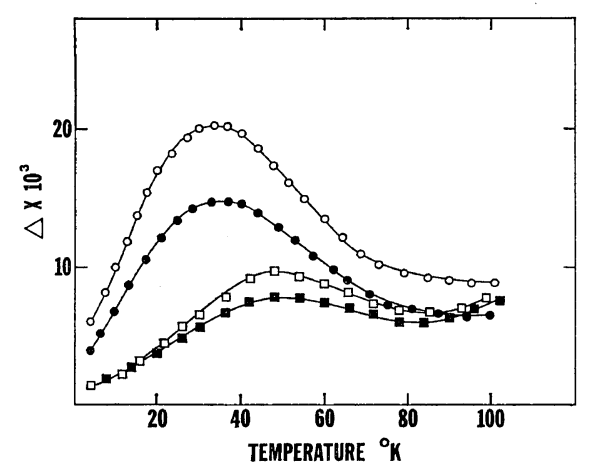

Figure 1. Effect of crystallinity on the $\delta$-relaxation in polystyrene and poly(p-xylylene): $\bigcirc$, amorphous PS; $\bullet$, crystalline PS; $\square$, crystalline PPX $(\rho=$ 1.110); $\boldsymbol{\square}$, crystalline PPX $(\rho=1.129) . \quad$ Ref 4 and 30. 
methylated polystyrenes. The intensity of the relaxation process depends on the number of groups participating in the motion, and decreases with increasing order in the solid state. This decrease in intensity is observed experimentally in both oriented ${ }^{6}$ and crystalline ${ }^{4}$ specimens.

A relaxation process similar to the $\delta$-process in PS is observed in poly ( $p$-xylylene) (PPX) and suggests that in some cases motions of in-chain phenyl rings are also active at cryogenic temperatures. This is apparent in Figure 1 where the behavior of two crystalline PPX samples is compared with that of amorphous and semicrystalline isotactic PS.

Recently, a second type of cryogenic relaxation process in linear polymers has been reported which can not be associated with motions of a specific chemical group. Armeniades and $\mathrm{Baer}^{8}$ observed two cryogenic loss peaks in poly(ethylene terephthalate) (PET), one at $26^{\circ} \mathrm{K}$ which was dependent on the level of crystallinity, and another at $46^{\circ} \mathrm{K}$ which was observed in oriented specimens. Neither relaxation was observed in the amorphous, unoriented polymer. Papir and Baer have observed similar relaxations in linear polyethylene $(\mathrm{PE})^{9}$ and poly(oxymethylene) $(\mathrm{POM}){ }^{10}$ This relaxation is termed the $\delta_{\mathrm{c}}$-relaxation to distinguish it from the cryogenic relaxations in PS and PPX. The principal features common to the $\delta_{\mathrm{c}}$-relaxation in all three polymers are summarized as follows.

(1) The $\delta_{\mathrm{c}}$-relaxation is essentially absent in specimens that are amorphous (PET) or have been rapidly quenched from the melt (PE and POM).

(2) The relaxation is observed in rolled or drawn specimens and increases in intensity as the amount of deformation increases (Figure 2).

(3) The relaxation is strongly dependent on the thermal history of the polymer. The intensity is observed to increase in oriented samples annealed under constraint, but decreases in oriented samples that are allowed to relax during annealing. Isotropic, quenched samples also exhibit a relaxation subsequent to annealing if the ends of the sample are held fixed during the heat treatment (Figure 3), but no
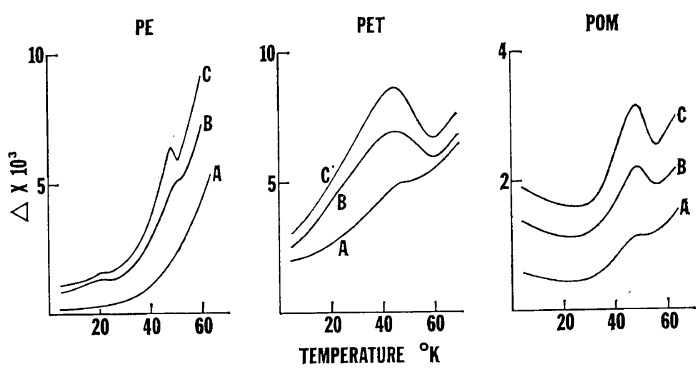

Figure 2. Effect of deformation on the $\delta_{\mathrm{c}}$-rexalation.

Polyethylene: A, undeformed ( $\rho=0.954)$; B, drawn $5.5 \times(\rho=0.963) ;$ C, drawn $8.5 \times(\rho=0.970)$.

Poly(ethylene terephthalate): A, undeformed ( $\rho=$ 1.331); B, drawn $3.5 \times(\rho=1.360) ; \mathrm{C}$, drawn $5.0 \times$ $(\rho=1.368)$.

Poly(oxymethylene): A, undeformed ( $\rho=1.403)$; B, rolled $5 \times(\rho=1.430) ; \mathrm{C}$, rolled $7 \times(\rho=1.434)$.

Ref 8-10.
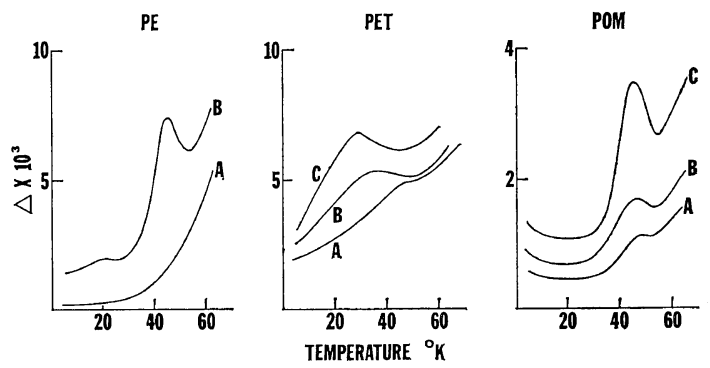

Figure 3. Effect of annealing on the $\delta_{\mathrm{c}}$-relaxation. Polyethylene: A $(\rho=0.954) ; \mathrm{B}(\rho=0.970)$.

Poly(ethylene terephthalate): A $(\rho=1.331) ;$ B ( $\rho=$ 1.337); C $(\rho=1.406)$.

Poly(oxymethylene): A $(\rho=1.403)$; B $(\rho=1.425)$; C $(\rho=1.440)$. Ref 8-10.

peak is observed if the polymer is unconstrained.

(4) For any polymer, the temperature of the $\delta_{\mathrm{c}}$-peak does not change with the amount of deformation.

(5) The $\delta_{\mathrm{c}}$-relaxation shows pronounced directional anisotropy. Samples with the torsion axis at $45^{\circ}$ to the orientation direction show a much stronger relaxation than those oriented at 0 or $90^{\circ}$ (Figure 4).

(6) In addition to the primary $\delta_{\mathrm{c}}$ peak at $45-50^{\circ} \mathrm{K}, \mathrm{PE}$ and POM both have secondary transitions at lower temperatures which 


\section{A. Hiltner and E. BAer}

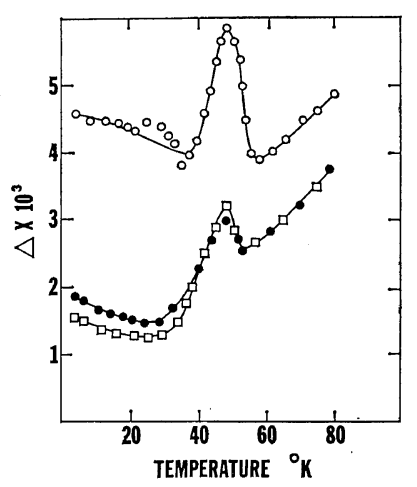

Figure 4. Anisotropy of the $\delta_{\mathrm{c}}$-relaxation in a POM sample rolled $7 \times(\rho=1.434)$, the angle indicated is between the torsion axis and the roll direction: $-0^{\circ} ; \bigcirc, 45^{\circ} ; \square, 90^{\circ}$. Ref 10 .

show the same dependence on orientation and heat treatment as the $\delta_{\mathrm{c}}$-peak.

(7) Although data on the temperature shift of the $\delta_{\mathrm{c}}$-peak with frequency is not available, the activation energy is estimated to be less than $4 \mathrm{kcal} / \mathrm{mol}$ using the empirical relationship between activation energy and the temperature of the relaxation maximum $(\mathrm{Hz}){ }^{11}$

(8) The process can not be characterized by a single relaxation time. The experimental peak is wider than that predicted from a single relaxation time and has a low temperature tail.

The complete dependence of these relaxation processes on the deformation and thermal history precludes a mechanism involving either specific group motions or motions at fold surfaces in the crystalline regions. Rather the data suggest that a mechanism involving irregularities within the ordered regions of the polymer should be sought.

Dislocations such as found in metals and other crystalline solids are also thought to exist in crystalline polymers. These may result from intrinsic point defects such as chain ends, impurities, isolated folds or branches. Predecki and Statton ${ }^{12}$ have considered possible molecular arrangements in the immediate vicinity of a chain end which produce dislocations. Strain energies of the various dislocations caused by chain ends have been calculated by Matsui,
Masui, and Wada. ${ }^{13}$ They predict that some screw dislocations may be more stable that the initial vacancy row. Motion of a dislocation under an applied stress is also considered by Predecki and Statton and Matsui, Masui and Wada. If dislocations can move under an applied stress, they will clearly affect mechanical properties including mechanical relaxations.

Several attempts have been made to explain transitions in PE in terms of defect mechanisms. Hoffman, et al., ${ }^{14}$ have suggested a mechanism proposed specifically for the $\gamma_{c}$-relaxation, which considers the reorientation of chains adjacent to the vacancy created by a chain end. Pechhold ${ }^{15}$ has suggested that chain conformations containing some gauche bonds in an otherwise trans chain are present in the crystalline regions of PE. He considers the $\gamma_{c}$-relaxation to arise from molecular rearrangement of blocks of $\operatorname{tgtg}^{\prime} t$ sequences. These blocks are similar to the screw dislocations proposed by Predecki and Statton. Although these models qualitatively account for many experimental features of the $\gamma$-process, NMR measurements of Olf and Peterlin ${ }^{16}$ could not be quantitatively described by either model. Recently, Matsui, Masui, and $\mathrm{Wada}^{13}$ have proposed a mechanism for the $\gamma_{c}$-relaxation in PE involving motions of dislocation over a Peierls barrier. Although the dislocations mechanism proposed for the $\gamma_{\mathrm{c}}$-relaxation can not be considered for the $\delta_{\mathrm{c}}$-process because of the high activation energies involved, the $\delta_{\mathrm{c}}$-motions may be a precursor to the higher temperature process.

\section{DISLOCATION MECHANISM}

Dislocation motions are known to give rise to relaxation processes in metals, and many characteristics of the $\delta_{c}$-peak in PE, POM, and PET are similar to experimental features of the Bordoni peaks observed at cryogenic temperatures in some fcc and bcc metals. The Bordoni peaks are thermally activated and occur at approximately the same temperature as the $\delta_{\mathrm{c}}$-peaks and hence have about the same activation energy, 2 to $4 \mathrm{kcal} / \mathrm{mol}$. They are also dependent on the process history of the metal, being most intense in cold worked samples, i.e., those having high dislocation densities, while annealing the 

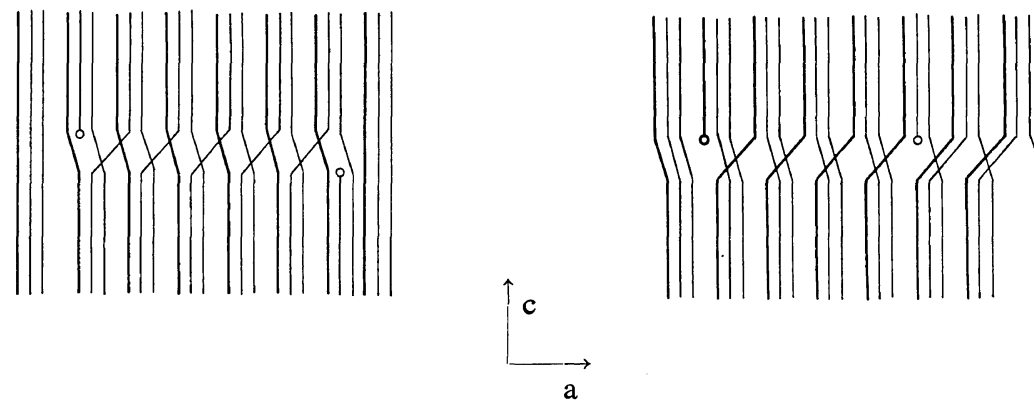

Figure 5. Two types of screw dislocation produced by a pair of chain ends in the same or adjacent planes.

metal to reduce the dislocation density decreases or eliminates the peak. However, like the $\delta_{\mathrm{c}^{-}}$ peak, the temperature of the Bordoni peak is essentially independent of the amount of cold work, which suggests that the activation energy is associated with motions of small segments of dislocations.

The proposed mechanism of the $\delta_{\mathrm{c}}$-relaxation is similar to that proposed by Brailsford ${ }^{19}$ for the Bordoni peaks in metals. The model for the $\delta_{\mathrm{c}}$-mechanism is based on the assumption that dislocations are present in crystalline polymers as a result of lattice deformation in the vicinity of local stresses. The local stresses are assumed to be immobile at low temperatures and hence act as pinning points for the dislocation.

On a molecular level, the presence of a localized stress inside the crystal lattice may cause the neighboring chain or chains to bend in such a way as to minimize the intra- and inter-chain

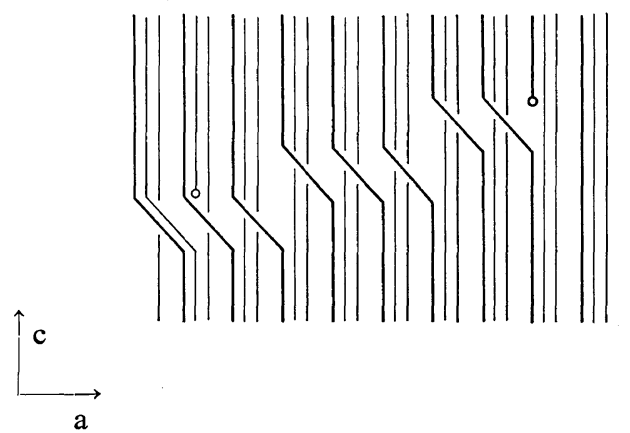

Figure 6. Screw dislocation having a Burgers vector with a component parallel to the chain direction. potentials. The bent chain segment is termed a jog, and the dislocation can be thought of as an ordered arrangement of jogged chain segments. For example, some screw dislocations which might be produced by chain ends in the crystal lattice are illustrated in Figure 5. The dislocation is preferentially oriented in one lattice plane with a Burgers vector perpendicular to the chain direction. A dislocation having a Burgers vector with a component in the chain direction can be formed if the jog is more pronounced in chains on one side of the dislocation than on the other. This lack of symmetry may result from the relative positions of the pinning points, as in Figure 6, or from nonuniform shear stresses. Discontinuities form where sections of the dislocation are displaced by one or more lattice spacings in the chain direction.
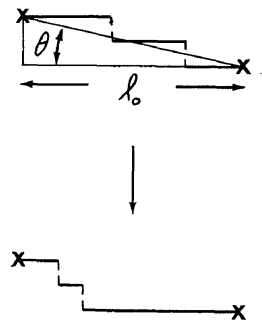

A

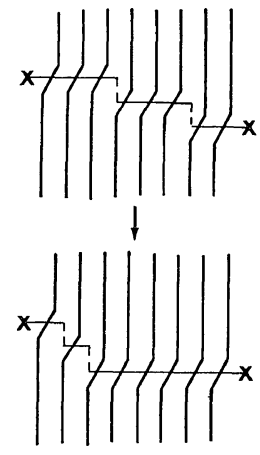

B
Figure 7. Redistribution of kinks along a dislocation: A, schematic representation; B, schematic including jogged chain segments. 


\section{A. Hiltner and E. Baer}

In this case the dislocation is assumed to be composed of a number of parallel dislocation segments each lying in a lattice plane and displaced laterally by one lattice spacing (Figure 7A). The discontinuity between dislocation segments is termed a kink. The kinks have an equilibrium distribution along the dislocation which corresponds to a minimum in the free energy. When an external stress is applied the distribution changes and since the process is thermally activated, gives rise to a relaxation. Movement of the kinks can be approximated by a two state model for the reorientation of the jogged chain segment at the edge of the kink. The jog is translated by the distance of one lattice spacing along the chain axis thus moving the kink one chain diameter along the $b$ axis (Figure 7B). Because the relaxation is controlled by the motion of a single jog, the activation energy, and hence the temperature at which the peak occurs, is an intrinsic property of the dislocation. Therefore parameters which depend on the pretreatment such as the density of kinks in the dislocation or the distance between pinning points, will not be expected to affect the temperature of the relaxation.

Only dislocations with a Burgers vector in the ac plane are considered above, although dislocations in other planes would also be expected. These dislocations may be responsible for the secondary peaks since jog conformations and the potential barriers to jog motion would be significantly different.

\section{RELAXATION INTENSITY}

The internal friction arising from diffusion of kinks along a pinned dislocation has been treated by Brailsford. ${ }^{19}$ Assuming a random distribution of kinks in the unstressed dislocation, the logarithmic decrement for a network with a dislocation density $N$ is given by

$$
\Delta=\pi N F(l, T) \frac{\omega \tau_{1}}{1+\omega^{2} \tau_{1}{ }^{2}} P(l) \mathrm{d} l
$$

where

$$
F(l, T)=\frac{8 G c^{2} B^{2} l^{3} n}{\pi^{4} k T}
$$

and $G$ is the shear modulus, $B$ the magnitude of the Burgers vector, $l$ the projection of the dislocation length on the $b$ axis, and the kink density $n=|\tan \theta| / c$. It is clear from eq 1 that the relaxation is not single process but a sum depending on the distribution of dislocation lengths $P(l)$. This is also seen experimentally in the peak breadth as compared to that predicted for a single relaxation process (Figure 8).

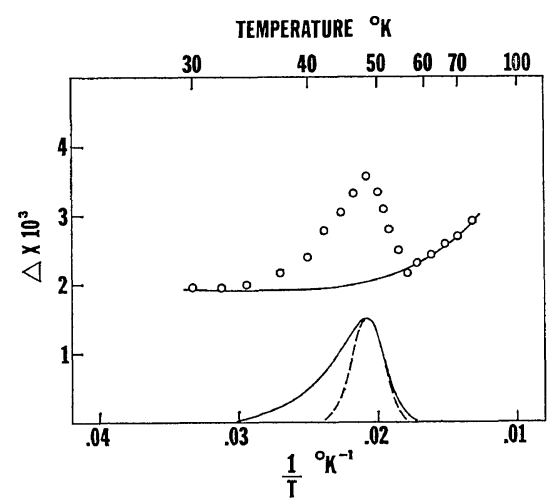

Figure 8. Shape of the $\delta_{\mathrm{c}}$-relaxation peak: $\bigcirc$, experimental points for a POM sample annealed with constraint ( $\rho=1.425)$ (ref 10$) ;-$, experimental curve with background damping subtracted; ---, calculated curve for a single relaxation time.

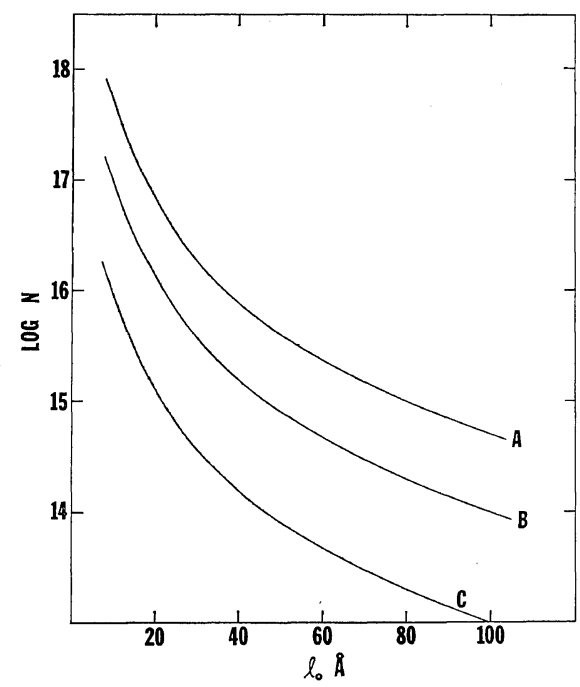

Figure 9. Relationship between $l_{0}$ the mean dislocation length and $N$ the dislocation density calculated for various values of the relaxation intensity: $\mathrm{A}, \Delta_{\max }=5 \times 10^{-3}$ (maximum observed); B, $\Delta_{\max }=10^{-3} ; \mathrm{C}, \Delta_{\max }=10^{-4}$ (experimental limit). 
If an exponential distribution with a mean dislocation length $l_{0}$ is assumed, the value of the logarithmic decrement at the peak maximum is given by

$$
\Delta_{\max }=2.1 N F\left(l_{0}, T_{0}\right)
$$

Using values of $G=10^{10} \mathrm{dyn} / \mathrm{cm}^{2}, c=2.5 \AA$ and $B=5 \AA$ for $\mathrm{PE}$, then

$$
\Delta_{\max } \simeq 50 N l_{0}{ }^{3}|\tan \theta|
$$

The intensity will thus be a function of three parameters: $N$ the dislocation density, $l_{0}$ the mean dislocation length, and $\theta$ which reflects the kink density in the dislocation. The intensity will clearly be very sensitive to $l_{0}$ and $N$ but not to $\tan \theta$ which changes by less than an order of magnitude for $10 \leq \theta \leq 40^{\circ}$. If $\theta$ is arbitrarily chosen the effect of $l_{0}$ and $N$ on the intensity can be examined. This is illustrated in Figure 9 where $N$ and $l_{0}$ are plotted for values of $\Delta_{\max }$ corresponding to the experimental limit, the maximum value of $\Delta$ observed and an intermediate value. If for example $l_{0}=40 \AA$, a dislocation density of $10^{14}$ dislocations/cc would not be detectable while a density of $10^{15}$ would produce an observable relaxation. It should be emphasized that $l$ refers to the distance between pinning points and if the dislocation contains more than 2 pinning points, $l$ may be considerably less than the apparent length of the dislocation.

\section{CHAIN CONFORMATION}

The conformation and motion of the jogged chain segment in the dislocation may be quite complex. A model involving tgtg' $^{\prime} t$ sequences in the PE chain has been suggested by Pechhold ${ }^{15}$ (Figure 10). Rotation of the bonds as indicated necessitates very slight extensions of the chain and results in translation of the jog by $2 \mathrm{CH}_{2}$ groups or one lattice spacing. The activation enegry for this process, neglecting neighboring chain effects, is calculated to be about $5 \mathrm{kcal} /$ mol. This is significantly higher than that obtained experimentally for the $\delta_{\mathrm{c}}$-process. However McMahon, et al., ${ }^{20}$ calculated much lower barriers for rotational motion of jogged chain segments if they assumed no a priori restrictions on the dihedral angles and expanded the jog

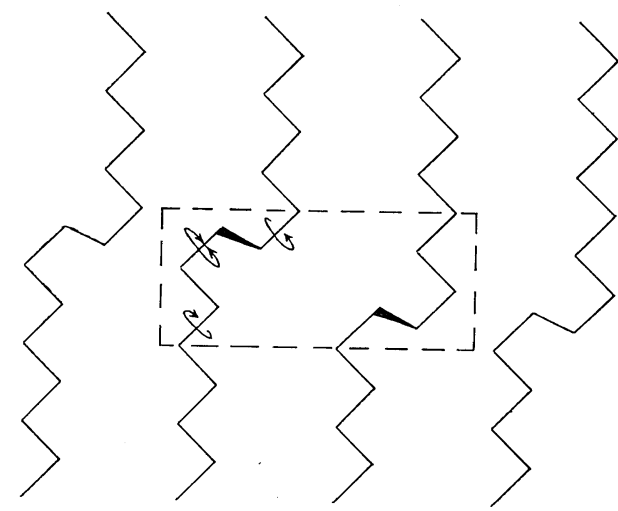

Figure 10. Translation of a jog in PE one lattice spacing along the chain direction.

from 3 to $7 \mathrm{CH}_{2}$ units. Matsui, Masui, and Wada have suggested that as may as $20 \mathbf{C H}_{2}$ units may be involved in the jog.

Unfortunately sophisticated potential calculations of defect conformations such as those of McMahon, et al., for PE are not available for POM and PET. POM is known to form a $9_{5}$ helix with the chain in a distorted gauche conformation. Local distortion of the helix has been suggested by Kedzie, ${ }^{21}$ who considers the local straightening out of the helix when one chemical repeat unit is removed, and by Papir and Baer $^{10}$ who consider the inclusion of extra units. One model involves a ...ggtg $g^{\prime}{ }^{\prime}$.. sequence and results in helix reversal. An alternative conformation is a gtg $^{\prime}{ }^{\prime} g$ sequence and, like the Kedzie defect, does not disturb the sign of the helix. More realistic conformations however probably include a longer chain segment with less distortion of the individual bonds than the gauche-trans transformation.

So far only linear polymers with very flexible backbones have been discussed and it remains to consider the effect of the bulky terephthalate group on the formation and motion of jogs in PET. The molecule has nearly planar zigzag conformation with the benzene ring in the plane of the zigzag. If the dihedral angles are restricted to trans or gauche, a reasonable jog conformation is shown in Figure 11 with two gauche C-O bonds. Translation of the jog clearly involves displacement of a benzene ring. This can be accomplished without rotation of the benzene 


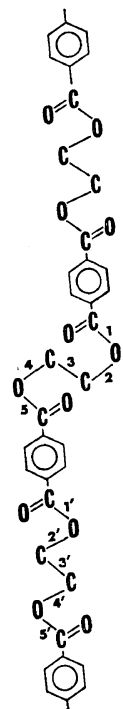

Figure 11. A jogged conformation of the PET chain.

ring by simultaneous rotation of bonds $2,4,2^{\prime}$ and $4^{\prime}$ or, more likely, by cooperative rotation and translation of all groups in the jog including the benzene ring. It should be recalled that relaxations in PS and PPX at these same temperatures have been attributed to phenyl group motions. Involvement of the large phenyl group may also be partially responsible for the larger relaxation strength of the $\delta_{\mathrm{c}}$-process in PET.

With the condition that only trans or gauche bonds are considered the model for PET suggests that a sequence of some minimum number of easily rotatable bonds is a prerequisite for formation and motion of a compact, low energy jog in a planar zigzag chain. It is of interest therefore to consider the cryogenic relaxation behavior of another polyester, poly (1,4-cyclohexylenedimethylene terephthalate) (PCT)<smiles>[3H]CCC1CCC(COC(=O)c2ccc(C(=O)OC(C)(C)C)cc2)CC1</smiles>

which contains sequences of only three flexible bonds. Little has been reported on the morphology of the polymer. X-ray studies by Boye ${ }^{22}$ show that, like PET, it crystallizes in a triclinic unit cell with the benzene rings very nearly parallel to the [100] plane. The cryogenic relaxation spectrum, in complete contrast to that of PET, shows a relatively strong $\delta$-relaxation in

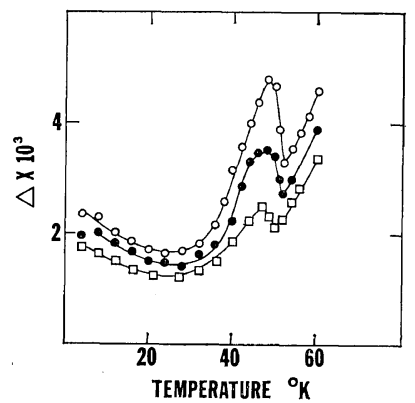

Figure 12. Effect on annealing on the $\delta$-relaxation in PCT: $\bigcirc(\rho=1.202) ; \quad(\rho=1.218) ; \square(\rho=1.225)$. Ref 23.

the amorphous polymer which decreases in intensity upon annealing (Figure 12). ${ }^{23}$ This relaxation is clearly associated with the disordered, amorphous regions and it is concluded that PCT does not exhibit a cryogenic relaxation process that can be associated with motion of defects in the ordered regions of the polymer. This can be attributed to the presence of the bulky cyclohexane ring in the PCT chain. The sequences of flexible bonds are not long enough for jogs to form that are able to participate in the low-energy conformational changes that give rise to the $\delta_{\mathrm{c}}$-relaxation.

\section{DEFORMATION AND ANNEALING}

The cryogenic relaxation behavior of the various polymers is summarized in Table I. As suggested previously, two types of relaxations can be distinguished: (1) the relatively broad, intense $\delta$-peaks such as observed in PS and PPX which have been attributed to molecular group motions, and (2) the less intense but very sharp $\delta_{\mathrm{c}}$-peaks observed in PE, POM, and PET. The $\delta_{\mathrm{c}}$-peaks are strongly dependent on the deformation and thermal history of the polymer. They are essentially absent in the quenched material but appear in oriented samples. Heat treatment increases the intensity if the ends of the sample are held fixed, but decreases it if the sample is allowed to relax.

It has been suggested that the $\delta_{\mathrm{c}}$-peak arises from redistribution of kinks along dislocations in ordered or crystalline regions of the polymer. The absence of the relaxation in quenched or 
Table I. Summary of cryogenic relaxations in polymers

\begin{tabular}{|c|c|c|c|c|}
\hline Polymer & Structure & Relaxations below $77^{\circ} \mathrm{K}$ & $\begin{array}{l}\Delta_{\max } \\
\times 10^{3}\end{array}$ & Ref \\
\hline Polystyrene (PS) & $-(-\mathrm{CHCH}$ & $40^{\circ} \mathrm{K}$ (broad) & 20 & 4 \\
\hline Poly(p-xylylene) (PPX) & & $50^{\circ} \mathrm{K}$ (broad) & 10 & 30 \\
\hline Linear polyethylene (PE) & $-\left(-\mathrm{CH}_{2} \mathrm{CH}_{2}\right.$ & $\begin{array}{l}48^{\circ} \mathrm{K} \text { (sharp) } \\
20^{\circ} \mathbf{K}\end{array}$ & $\begin{array}{l}3 \\
0.5\end{array}$ & 9 \\
\hline Poly(oxymethylene) (POM) & $-\left(-\mathrm{CH}_{2} \mathrm{O}-\right.$ & $\begin{array}{l}45^{\circ} \mathrm{K} \text { (sharp) } \\
<4^{\circ} \mathrm{K}\end{array}$ & 1.5 & 10 \\
\hline $\begin{array}{r}\text { Poly(ethylene terephthalate) } \\
\text { (PET) }\end{array}$ & & $\begin{array}{r}\text { Oriented } 48^{\circ} \mathrm{K} \text { (sharp) } \\
\text { Crystalline } 26^{\circ} \mathrm{K} \text { (sharp) }\end{array}$ & $\begin{array}{l}4 \\
2.5\end{array}$ & 8 \\
\hline
\end{tabular}

amorphous material implies that dislocations are not present in significant numbers either because of low amounts of crystallinity (PET) or because the rapid cooling does not permit formation of a regularized array of jogged chain segments (PE and POM). It follows that the existence of dislocations in oriented polymers is intimately related to the mechanism of deformation.

A model for the deformation of chain-folded crystalline polymers has been proposed by Peterlin. $^{24}$ Although based primarily on work with polyethylene and polypropylene, the observations of O'Leary and Geil ${ }^{25}$ and Gezovich and $\mathrm{Geil}^{26}$ on the deformation of POM are also in good agreement with the model. Initially, blocks of folded chains tilt into the draw direction. With further deformation the blocks of folded chains break off and, together with unfolded chain sections resulting from such a break, are incorporated into a fiber forming irregular lamallae oriented normal to the draw direction. The fibers are interconnected by tie molecules which may either have been present in the starting material or are produced by chain unfolding during the necking process. When further deformation occurs, the fibers slide past one another and the interfibrillar tie molecules become highly strained.

As a result of drawing, the lamellae in the fiber structure show a high concentration of imperfections. The low density of the crystalline regions of drawn polymers, infered from the decreased intensity of the X-ray long spacing

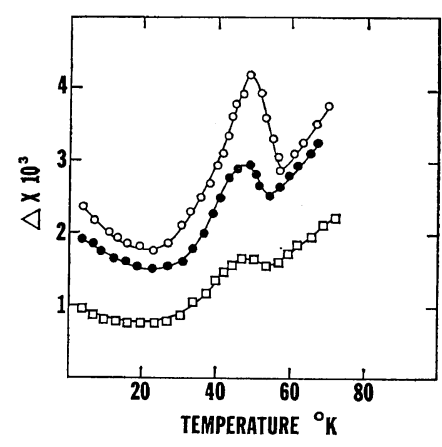

Figure 13. Effect of annealing conditions on the $\delta_{\mathrm{c}}$-relaxation in oriented POM: 0 , rolled $7 \times(\rho=$ 1.434); $\bigcirc$, rolled and subsequently annealed with constraint ( $\rho=1.444) ; \square$, rolled and annealed without constraint $(\rho=1.442)$. Ref 10 .

reflection, is generally attributed to the high concentration of these imperfections. If the deformed polymer is subsequently annealed at temperatures where the polymer chains are known to have translational mobility, the density of the crystalline regions increases as the order is perfected. If the $\delta_{\mathrm{c}}$-relaxation is a manifestation of the imperfections introduced during deformation, the decrease in intensity when the polymer is annealed is consistent. One of the most important experimental features of the $\delta_{\mathrm{c}}$ relaxation, however, is the tremendous sensitivity to constraint during annealing either with or without prior deformation (Figure 13). Recent work by Balta-Calleja and Peterlin ${ }^{27}$ suggests this can 


\section{A. Hiltner and E. BAer}

not be attributed to an inhibition of molecular mobility under conditions of constraint. They observed that for both deformed and undeformed polypropylene films the molecular mobility, as reflected by the rate of lamellar thickening, was the same irregardless of whether the ends of the film were held fixed or not. They suggest that when the temperature is sufficiently high to permit longitudinal chain mobility, the lattice imperfections heal nearly instantaneously. On the other hand, the mechanism responsible for disorientation and shrinkage in the unconstrained samples, processes experimentally found to be inhibited by constraint, is not intimately connected with lamellar thickening beyond the condition of chain mobility. Here the important morphological units are the inter- and intra-fibrillar tie molecules. Tie molecules anchored in different crystals, upon sufficient mobilization, try to be included in the crystal lattice. Driven by this refolding tendency of the tie molecules, the sample returns almost to the original morphology. If, however, the annealing is performed with fixed ends so that the sample can not shrink, interfibrillar tie molecules can only relax by partial crystallization or assumption of an amorphous conformation in the non crystalline regions. Such adjustments, necessitating a lengthening of the tie molecules, impose stresses on the crystalline blocks to which the tie molecule is anchored similar to the stresses imposed during deformation. These stresses can be relieved by chain unfolding or by the formation of dislocations in the crystalline blocks. Therefore it is suggested here that some dislocations are already present in the drawn material as a result of the stress imposed on the tie molecules during deformation. The number of strained tie molecules and hence the number of dislocations is known to increase with increasing draw ratio. As long as the film is under constraint, annealing can only result in the creation of new dislocations and the perfection of those present. Only when the constraint is removed can the tie molecules relax and either become incorporated as part of the lattice or assume some other more stable configuration.

The same arguments. apply to interlamellar tie molecules in the undeformed material. When the isotropic samples is annealed with fixed ends, the tie molecules are unable to relax with the surrounding lamellar material and stresses are set up in the region where the tie molecule is anchored.

Peterlin's model for the deformation of semicrystalline polymers does not apply directly to glassy polymers such as PET. The deformation of this polymer is the subject of an extensive study by Yeh and Geil. ${ }^{28}$ When glassy PET is drawn, strain-induced crystallization occurs during the elongation. Contrary to the general impression that the amorphous state consists of entangled, randomly coiled molecules, glassy PET consists of small clusters of aligned chain segments. One molecule may easily be incorporated into several clusters forming a potential tie molecule. When the glassy polymer is drawn the clusters retain their identity but become aligned in rows at an angle to the draw direction. Upon annealing these rows merge to form lamellae. Yeh and Geil have suggested a modified fringed-micelle model for the glassy polymer in which the ordered clusters are the "crystallites." In the undrawn polymer the molecules in the clusters are aligned but are not necessarily packed in a three-dimensional lattice. Colddrawing improves intracluster order as well as intercluster order, as evidenced by the highly ordered X-ray fiber patterns obtained from colddrawn specimens. This model of drawn PET contains the elements necessary for the formation of dislocations, specifically blocks of highly ordered chain segments linked by tie molecules

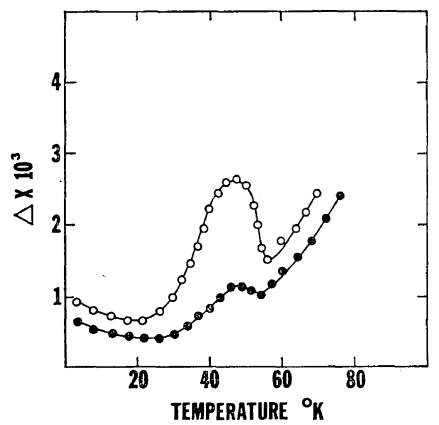

Figure 14. Effect of a comonomer on the $\delta_{\mathrm{c}}$-relaxation: - POM homopolymer $(\rho=1.418)$; $\bigcirc$, POM with $1.4-\%$ ethylene oxide units $(\rho=1.417)$. Ref 10. 
through which external stresses can be transferred to the crystalline blocks.

Although the most important experimental features of the $\delta_{\mathrm{c}}$-relaxation have been accounted for, the effect of both crystallization conditions and the presence of a comonomer such as a small amount of PEO polymerized with POM (Figure 14), suggest that there may be other stresses on the lattice which can not be relieved by lattice mobility and also result in dislcations. These may involve the presence of a comonomer unit in the lattice ${ }^{29}$ or the effect of temperature gradients on the crystallization process during slow-cooling.

\section{CONCLUSION}

It has been shown that dislocations of the type likely to occur in polymer crystals can move under an applied stress and should affect mechanical properties including internal friction. Moreover it is suggested that pretreatments such as deformation or annealing under constraint are especially conducive to the formation of extensive dislocation networks, and therefore identify motions of these dislocations as being responsible for the $\delta_{\mathrm{c}}$-relaxation.

Because these motions occur at very low temperatures, they may be precursors to higher temperature relaxations such as the $\gamma$-processes in PE and POM. The mechanisms suggested by Matsui, Masui and Wada, ${ }^{13}$ and Papir and Baer $^{10}$ involving motion of a dislocation over a Peierls barrier would be consistent with this view. In addition, the presence of mobile dislocations in a polymeric solid should significantly affect the macroscopic deformation properties. The influence of these dislocations should be especially important at cryogenic temperatures where other stress transfer mechanisms are frozen out.

Finally, it should be noted that while the kink diffusion mechanism proposed above is consistent with the available data, we do not suggest that it is conclusive, and modifications may be expected as a result of work currently in progress.

Acknowledgment. This research was generously supported by the Atomic Energy Commission,
University of California Lawrence Radiation Laboratory.

\section{REFERENCES}

1. N. G. McCrum, B. F. Read, and G. Williams, "Anelastic and Dielectric Effects in Polymeric Solids," John Wiley and Sons, New York, N. Y., 1967.

2. J. M. Crissman, J. A. Sauer, and A. E. Woodward, J. Polym. Sci., Part A, 2, 5075 (1964).

3. K. M. Sinnott, J. Appl. Phys., 29, 1433 (1958).

4. C. D. Armeniades, E. Baer, and J. K. Rieke, J. Appl. Polym. Sci., 14, 2635 (1970).

5. J. M. McCammon, J. A. Sauer, and R. N. Work, J. Polym. Sci., Part A-2, 7, 1721 (1969).

6. V. Frosini and A. E. Woodward, ibid., Part $A-2,7,525$ (1969).

7. J. M. Crissman, A. E. Woodward, and J. A. Sauer, ibid., Part A, 3, 2693 (1965).

8. C. D. Armeniades and E. Baer, ibid., Part $A-2$, 9, 1345 (1971).

9. Y.S. Papir and E. Baer, J. Appl. Phys., in press.

10. Y. S. Papir and E. Baer, Matl. Sci. Eng., in press.

11. T. E. Stephenson, Trans. Met. Soc. AIME, 233, 1183 (1965).

12. P. Predecki and W. O. Statton, J. Appl. Phys., 37, 4053 (1966); 38, 4140 (1967).

13. M. Matsui, R. Masui, and Y. Wada, Polymer J., 2, 134 (1971).

14. J. D. Hoffman, G. Williams, and E. Passaglia, J. Polym. Sci., Part C, 14, 173 (1965).

15. W. Pechhold and S. Blasenbrey, Kolloid Z., 216/217, 235 (1967); W. Pechhold, ibid., 228, 1 (1968); H. Scherr, W. Pechhold, and S. Blasenbrey, Kolloid Z. Z. Polym., 238, 396 (1970).

16. H. G. Olf and A. Peterlin, J. Polym. Sci., Part A-2, 8, 753, 771, 790 (1970).

17. D. H. Niblett and J. Wilks, Adv. Phys., 9, 1 (1960).

18. R. Gibala, M. K. Korenko, M. F. Amateau, and T. E. Mitchell, J. Phys. Chem. Solids, 31, 1889 (1970).

19. A. D. Brailsford, Phys. Rev., 122, 778 (1961).

20. P. E. McMahon, R. C. McCullough, and A. A. Schlegel, J. Appl., Phys., 38, 4123 (1967).

21. P. H. Geil, "Polymer Single Crystals," Interscience, New York, N. Y., 1963, p 338.

22. C. A. Boye, J. Polym. Sci., 55, 275 (1961).

23. A. Hiltner and E. Baer, J. Macromol. Sci.- 


\section{A. Hiltner and E. Baer}

Phys., B, in press.

24. A. Peterlin, "Man-Made Fibers,"' Vol. 1, Mark, Atlas, and Cernia, Ed., Wiley-Interscience, New York, N. Y., 1967, Chap. 8.

25. K. O'Leary and P. H. Geil, J. Macromol. Sci.Phys., B1, 147 (1967).

26. D. Gezovich and P. H. Geil, J. Macromol. Sci.Phys., B, in press.
27. F. J. Balta-Calleja and A. Peterlin, J. Macromol. Sci._Phys., B4, 519 (1970); Makromol. Chem., 141, 91 (1971).

28. G. S. Y. Yeh and P. H. Geil, J. Macromol. Sci.-Phys., B1, 235, 251 (1967).

29. C. A. Garber and P.H. Geil, Makromol. Chem., 113, 236 (1968).

30. A. Hiltner and E. Baer, unpublished results. 